\title{
Synthesis and Structure of Complexes of Acyl N-Aminides with Zinc(II) Salts
}

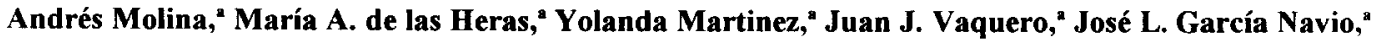 \\ Julio Alvarez-Builla, * Pilar Gomez-Sal, ${ }^{\text {b }}$ Rosario Torres ${ }^{c}$ \\ Departamento de Quimica Orgánica, ${ }^{a}$ Quimica Inorgánicab and Unidad Central de Servicios Analiticos (UCSA), Universidad de Alcala, \\ 28871-Alcalá de Henares, Madrid. Spain
}

\begin{abstract}
The structures of complexes obtained by reacting acyl $\mathrm{N}$-aminides and $\mathrm{ZnCl}_{2}$ were ascertained by $\mathrm{X}$ ray analysis and were show to incorporate one $\mathrm{Zn}$ atom and two molecules of the $\mathrm{N}$-aminide in a distorted tetrahedral geometry. Bis-aminides act as bidentate ligands forming $\mathrm{ZnLCl}_{2}$ complexes.

(C) 1997 Elsevier Science Ltd.
\end{abstract}

We recently reported on the synthetic utility of $\mathrm{N}$-aminides $1^{1}$ which, upon reaction with $\mathrm{CrO}_{3}$ can be converted into selective oxidising reagents $\mathbf{2}^{2}$ or when alkylated afford selective acylating reagents 3 which react with organometallics and DIBALH producing ketones and aldehydes in good to excellent yields. ${ }^{3-5}$<smiles></smiles>

1<smiles>[R]C(=O)N[n+]1ccn(C)c1</smiles>

2<smiles>[R]C(=O)N(C)[n+]1ccn(C)c1</smiles>

3

\section{Scheme 1}

Our interest in the chemical properties of these heterobetaines ${ }^{1 \mathrm{c}}$ led us to explore the application of some acyl $\mathrm{N}$-aminides as potentials dienes in the Diels-Alder reaction. Although our efforts were all unsuccessful we observed that in one of the attempted reactions, the zinc chloride used as catalyst reacted with the $\mathrm{N}$-cinnamoyl aminide forming a very stable complex with a stoichiometry $\mathrm{ZnL}_{2} \mathrm{Cl}_{2}$. Although the result was not unexpected since salts of Cobalt(II), Copper(II), Palladium(II), Platinum(II), Rhodium(III) and Iridium(III) have been reported to react with benzoyl $\mathrm{N}$-aminides to afford products containing betaines metallated on the ortho position of aryl moiety, ${ }^{6,7}$ in this case the $o$-metallation seemed to be less probable as such co-ordination would imply a seven membered ring.

When the benzoyl $\mathrm{N}$-aminide was tested as ligand instead of cinnamoyl $\mathrm{N}$-aminide, no evidence that the phenyl ring had been metallated in a position ortho to the carbonyl group was observed, and so we decide to unambigously 
establish the structure of these complexes by X-ray analysis. The results of this study are reported herein along with the structure of complexes obtained from the reaction of bis-aminides (bidentate ligands) with $\mathrm{Zn}$ (II) salts.

Complexes 4 were cleanly and rapidly obtained in good yields by reacting of aminides 1 with $\mathrm{ZnCl}_{2}$ in dichloromethane. The complexes can also be formed in other solvents such as ethanol, although in some cases the reaction seems to be slower. All complexes are of a white colour and are stable in aqueous solution. As expected, the free betaine can be easily regenerated by treatment of the complex with common bases.

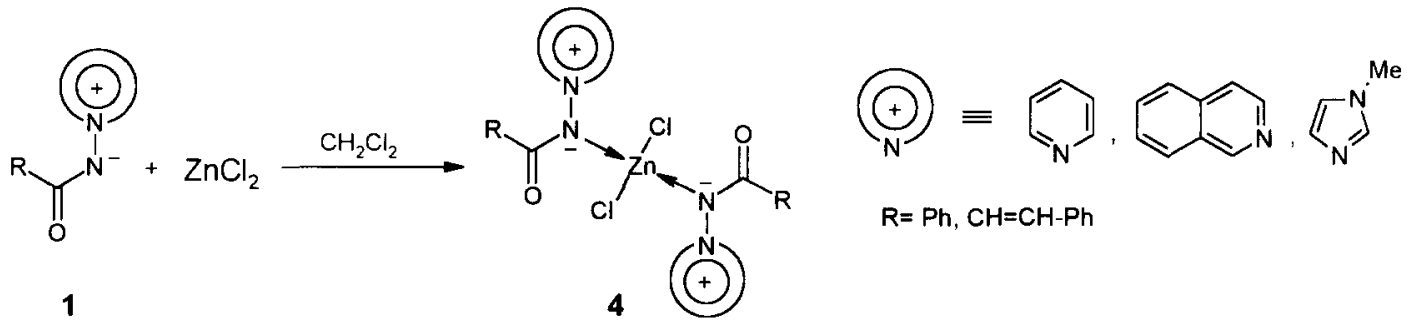

Scheme 2

The infrared spectra of these complexes show characteristic changes due to co-ordination of $\mathrm{Zn}$ with the nitrogen atoms, in particular the band corresponding to the carbonyl group, which in the complexes appears in the range 1642-1684 cm $\mathrm{cm}^{-1}$ while in the precursor it shows up at a lower frequency ranged of $1566-1653 \mathrm{~cm}^{-1}$ as a result of the contribution of the imidate resonance form. The localization of the negative charge on the nitrogen as a consequence of co-ordination to the metal enhances the double bond character of the carbonyl group, leading to a considerable shift to higher absorption frequencies with values closely approaching those found for $\mathrm{N}$-methyl- $\mathrm{N}$ cycloiminium amides (1652-1701 cm comparing the 'H NMR spectra of the aminides with those of the complexes, the protons of the azinium moieties usually appeared deshielded in complexes bearing the benzoyl moiety ( $\mathbf{4 b}$ and $\mathbf{4 d}$ ) whereas in those containing the cinnamoyl substituent ( $\mathbf{4 a}$ and $\mathbf{4 c}$ ) the protons of the azinium rings appear at very similar chemical shifts in both the free aminide and the complex. On the other hand, in the imidazolium complexes $4 \mathbf{e}$ and $\mathbf{4 f}$ the $\mathrm{C}-2$ protons of the imidazolium ring appear shielded, with the effect being most pronunced in $4 \mathbf{e}(0.65 \mathrm{ppm})$ than in $\mathbf{4 f}(0.02 \mathrm{ppm})$ The deshielding of the heteroaromatic protons in the complexes are consistent with co-ordination to the nitrogen atom which restricts delocalization of the negative charge over the pyridinium moiety. The apparent anomalous shielding observed for protons in $\mathbf{4 e}$ and $\mathbf{4 f}$ is likely the result of the stronger influence of the acyl substituent in these complexes which shifts them to highfield relative to the aminides

The structure of these complexes was established by X-ray analysis of the complex $4 \mathbf{a}$, which showed the best crystalline properties. In the asymmetric unit of the unit cell two crystallographic independent but chemically equivalent molecules appear. The two molecules are related by a pseudo-center of symmetry, and the differences between them are small. As expected, the metal appears co-ordinated to two organic ligands through the ylidic nitrogen atoms and surrounded by the two chloro atoms, adopting a distorted tetrahedral geometry. The largest angular deviation from a tetrahedral is $100.9(1)^{\circ}$ for $\mathrm{Cl1}-\mathrm{Znl}-\mathrm{N} 2$. The $\mathrm{Zn}-\mathrm{Cl}$ bond distances range from $2.246(2)$ to $2.262(2) \AA$ and the $\mathrm{Zn}-\mathrm{N}$ distances range from 2.022(4) to 2.047(5) $\AA$. The two betaine ligands bonded to the same $\mathrm{Zn}$ atom are similar except for the angle between the main plane of the pyridinium ring and the cinnamoyl moieties which are $79.8(2)^{\circ}$ for the system with $\mathrm{N} 1$ and $\mathrm{N} 2$ and $58.9(2)^{\circ}$ for the system with $\mathrm{N} 3$ and $\mathrm{N} 4\left(\left(79.12(2)^{\circ}\right.\right.$ and $47.03(2)^{\circ}$ are the values for the second molecule). The bond distances between the oxygen and the carbon atoms 
of the carbonyl groups range from $1.208(7) \AA$ to 1.249 (7) $\AA$, confirming the double bond character of these bonds. Figure 1 shows the structure of $\mathbf{4 a}$ and Figure 2 its unit cell packing.

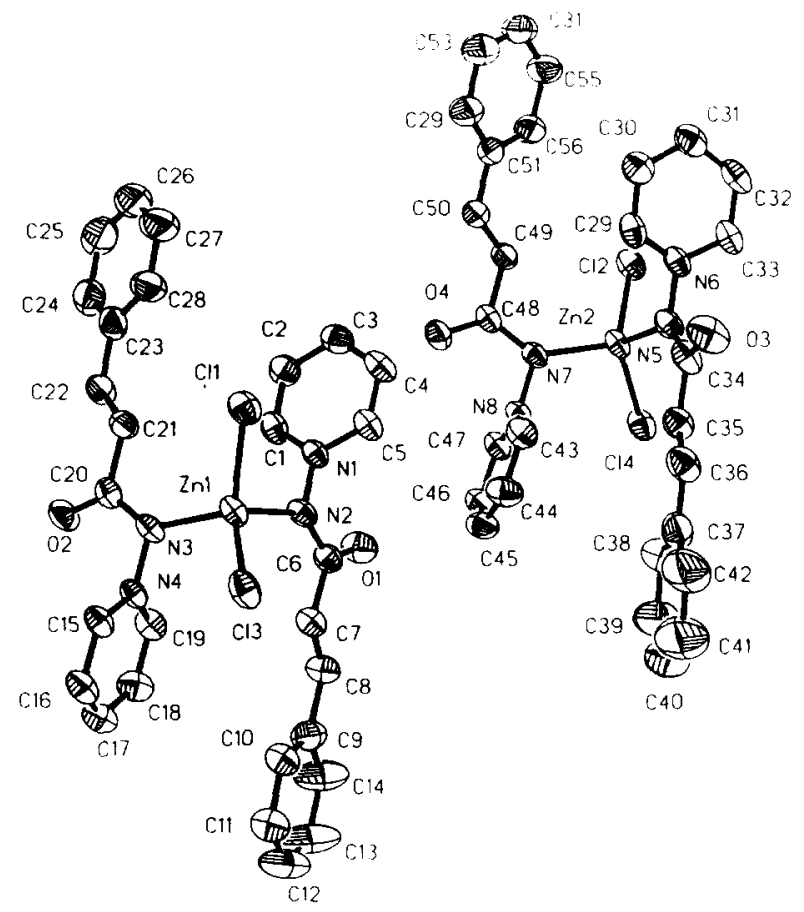

Figure 1. ORTEP drawing of compound $4 \mathbf{a}$ with atomic numbering scheme.

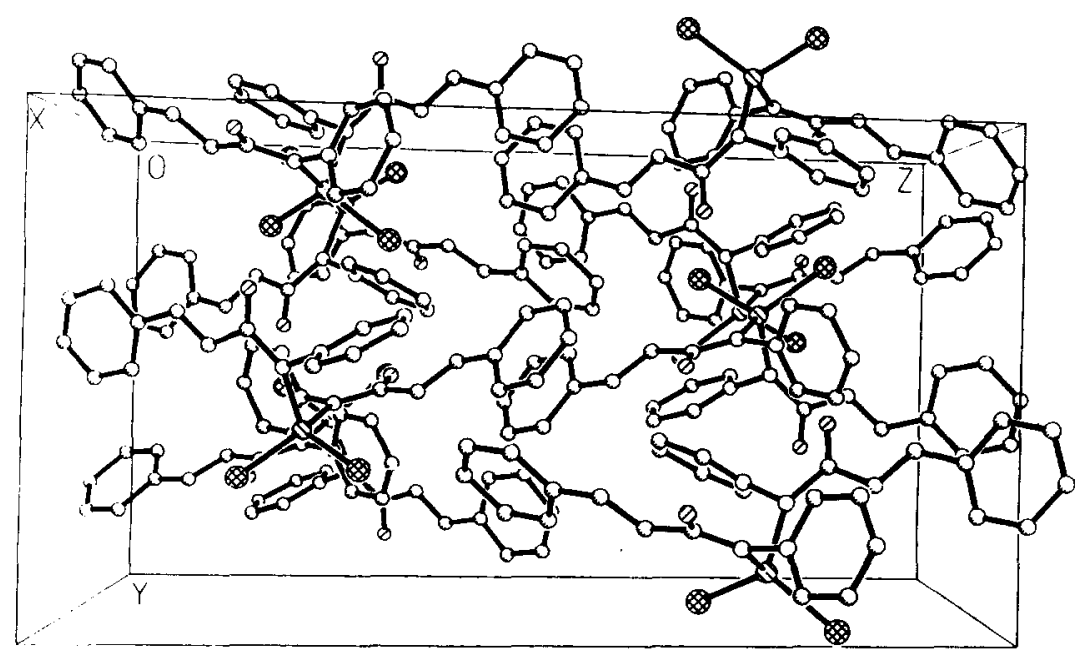

Figure 2. Unit cell packing of complex $4 \mathbf{a}$. 
Table. Crystal data and structure refinement of $\mathbf{4 a}$.

\begin{tabular}{|c|c|c|c|}
\hline $\begin{array}{l}\text { Empirical formula } \\
\text { Formula weight }\end{array}$ & $\begin{array}{l}\mathrm{C}_{28} \mathrm{H}_{24} \mathrm{~N}_{4} \mathrm{O}_{2} \mathrm{Cl}_{2} \mathrm{Zn}_{2} \\
584.8\end{array}$ & $\begin{array}{l}\text { Absorption coefficient } \\
F(000)\end{array}$ & $\begin{array}{l}11.36 \mathrm{~cm}^{-1} \\
2400\end{array}$ \\
\hline Temperature & $293(2) \mathrm{K}$ & Crystal size & $0.25 \times 0.35 \times 0.30$ \\
\hline Wavelength & 0.71069 & $\theta$ range for data collection & $-21<\mathrm{H}<21, \quad 0<\mathrm{K}<15$, \\
\hline Crystal system & Monoclinic & & $0<\mathbf{L}<28$ \\
\hline Space group & $\mathrm{P} 21 / \mathrm{c}$ & Reflections collected & 8044 \\
\hline \multirow[t]{4}{*}{ Unit cell dimensions } & $a=18.175(4) \AA$ & Independent/observed & $7602(2 \sigma \mathrm{I}$ criterium $)$ \\
\hline & $\mathrm{b}=12.874(5) \AA$ & Refinement method & Full-matrix least- \\
\hline & $c=23.821(4) \AA$ & & squares on $F$ \\
\hline & $\beta=98.06(3)$ & Data/restraints/parameters & $7602 / 0 / 448$ \\
\hline Volume & $5519(4) \AA$ & Final R indices $[(I-2(I)]$ & $R \mathbf{R}=0.072, R_{w}=0.098$ \\
\hline$Z$ & 8 & Largest diff. peak and hole & 0.851 and $-1.109 \mathrm{eA}^{-3}$ \\
\hline Density (calculated) & $1.408 \mathrm{~g} / \mathrm{cm}^{3}$ & & \\
\hline
\end{tabular}

As with aminides 4 , we also tested the ability of some thio analogs 5 to acts as ligands. For example, we prepared the thioaminide $5 \mathrm{a}$ by reacting $4 \mathrm{a}$ with $\mathrm{P}_{4} \mathrm{~S}_{10}$ in the presence of triethylamine. ${ }^{8}$ However, after treatment of $5 \mathbf{a}$ with $\mathrm{ZnCl}_{2} 4 \mathbf{a}$ was invariably recovered unalterated even on heating at reflux.<smiles>Cc1ccccc1</smiles>

$4 a$

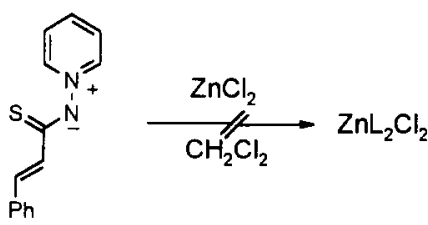

5a

Scheme 3

Once the structures of complexes 4 was established we reasoned that bis-aminides $6^{4,9}$ could behave as bidentate ligands to possibly give either the $1: 1$ complexes 7 or complexes 8 which involve the more unusual $2: 2$ mode of co-ordination. ${ }^{10,11}$<smiles>O=C1[Y]C(=O)[C@@](Cl)(n2cccc2)C(Cl)(Cl)N1n1cccc1</smiles>

7

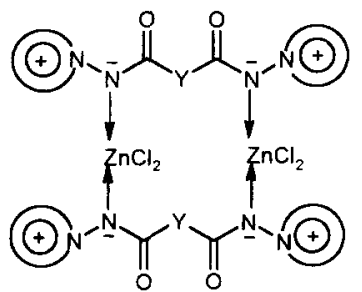

Scheme 4 
The bis-aminides were prepared as previously described either from the reaction of dicarboxylic acids or diacyl dihalides with $\mathrm{N}$-aminopyridinium mesitylene sulfonate. ${ }^{4}$ When the bis-aminides 6 , in which the two aminide moieties were linked by a flexible polymethylene chain, were reacted with $\mathrm{ZnCl}_{2}$ in $\mathrm{CH}_{2} \mathrm{Cl}_{2}$ the complexes obtained showed physical and spectroscopic characteristics similar to those found for 4 . However, in this case we were unable to obtain suitable crystals for $\mathrm{X}$-ray analysis and the structure 7 assigned to these complexes was established on the basis of analytical and spectroscopic data, in particular mass spectrometry (FAB-MS). In the infrared spectra of these complexes the carbonyl groups also appeared at higher wavelengths than in the corresponding bis-aminides although the shifts in frequencies were less significant than in complexes 4 since the carbonyls of these bis-aminides appeared in the range $1615-1620 \mathrm{~cm}^{-1}$

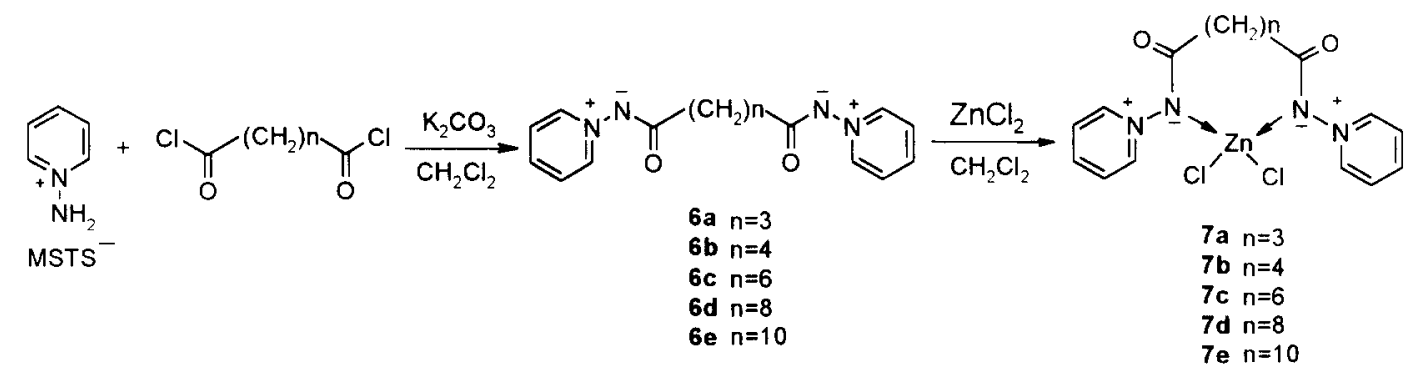

\section{Scheme 5}

In the 'H NMR of these complexes the chemical shifts of the pyridinium protons when compared to those of the corresponding protons in the bis-aminides show a similar pattern to that found for complexes 4 . Further evidence for nitrogen co-ordination was obtained when it was unsuccessfully attempted to double $\mathrm{N}$-methylate complex $7 \mathbf{e}$ while the corresponding aminide $6 \mathbf{e}$ could easily be transformed into the bis-salt 9 by reacting with methyl iodide at room temperature.

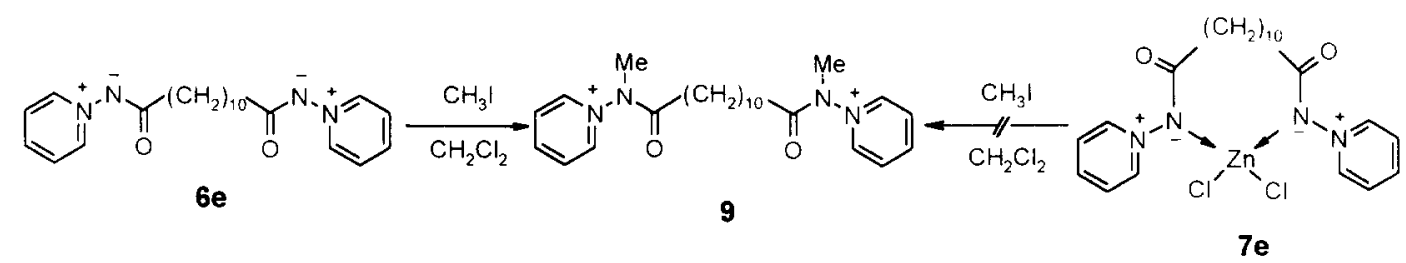

\section{Scheme 6}

When compound 7e, taken as representative of this series was subjected to FAB-MS in positive-ion spectrum the higher mass peak in the spectrum appeared at $m / z=519(2 \%)$ with two significant peaks at $m z=483(36 \%)$ and $m z=383(78 \%)$ and were assigned as corresponding to $\left(\mathrm{C}_{22} \mathrm{H}_{30} \mathrm{Cl}_{2} \mathrm{~N}_{4} \mathrm{O}_{2} \mathrm{Zn}\right) \mathrm{H}^{+}$, to the loss of chloride $(\mathrm{MH}-\mathrm{Cl})$ and to the loss of $\mathrm{ZnCl}_{2}\left(\mathrm{MH}^{+}-\mathrm{Cl} \mathrm{Zn}\right)$ respectively. This result seems to lend support to structure 7 for these complexes assuming that a similar fragmentation pattern for the $2: 2$ complex would afford a distribution of peaks clearly showing evidence for the structure 8 . The positive-ion FAB-MS of compounds $7 \mathbf{b}$ and $7 \mathbf{c}$ also showed a similar fragmentation pattern with the common fragments peaks for $7 \mathrm{~b}$ observed at $m / z=435\left(\mathrm{C}_{16} \mathrm{H}_{18} \mathrm{Cl}_{2} \mathrm{~N}_{4} \mathrm{O}_{2} \mathrm{ZnH}, 1 \%\right)$, $399(12 \%)$ and $299(100 \%)$ and for $7 \mathrm{c}$ at $m / z=463\left(\mathrm{C}_{18} \mathrm{H}_{22} \mathrm{Cl}_{2} \mathrm{~N}_{4} \mathrm{O}_{2} \mathrm{ZnH}^{+}, 9 \%\right), 427(67 \%)$ and $327(100 \%)$. 
In an attempt to form the complexes with the $2: 2$ stoichiometry we were ble to prepare the bis-aminide $6 f$ in which the two nitrogen atoms are joined by a rigid linker, which would prevent the formation of complexes with the 1:1 stoichiometry. This bis-aminide was unalterated in the presence of $\mathrm{ZnCl}_{2}$ under similar or stronger conditions than those employed for the preparation of 7 . The result can be assumed as a further support for the structure assigned to complexes 7

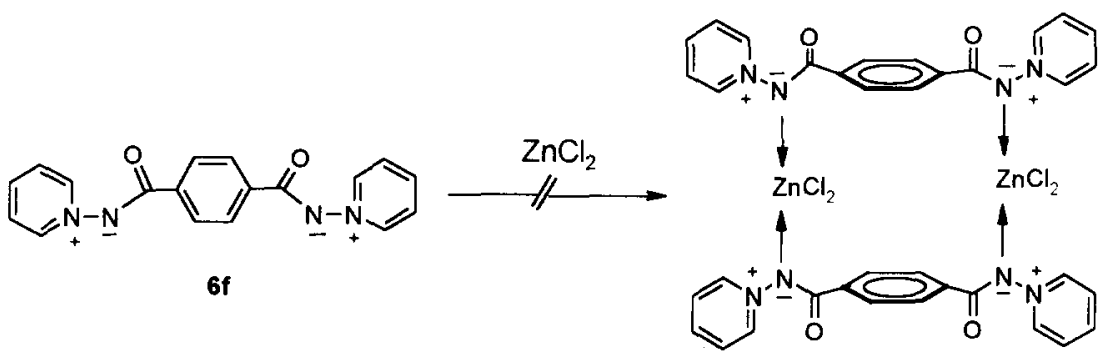

Scheme 7

In conclusion, this work has shown that the reaction of acyl $\mathrm{N}$-aminides and $\mathrm{Zn}$ (II) easily affords $\mathrm{ZnL}_{2} \mathrm{Cl}_{2}$ complexes, the structures of which were established by X-ray analysis. Attempts to form complexes with the $2: 2$ stoichiometry by using flexible bis-aminides as bidentate ligands were unsuccessful, as these ligands tended to give the $1: 1$ complexes. Moreover, at this point in time the evidence suggests that a rigid bis-aminide unable to give the $1: 1$ complex is also incapable of forming $2: 2$ type complexes.

\section{Experimental}

General Procedures. Melting points were determined on a Buchi SMP-20 apparatus and are uncorrected. ${ }^{1} \mathrm{H}$ NMR were recorded on a Varian Unity 300 spectrometer and were referenced to TMS. IR spectra were obtained on a Perkin-Elmer 1310 spectrophotometer. Microanalyses were performed on a Heraeus CHN Rapid analyzer and MS were obtained on a Hewlett-Packard 5988 A spectrometer. Positive FAB-MS mass spectra were recorded using 3-nitrobenzylic alcohol matrix on a VG AutoSpec mass spectrometer. Chromatography was performed on silica gel 60 (230-400 meshs). All reagents were obtained from commercial sources and were used as adquired. Solvents were dried before using. Acyl $\mathrm{N}$-aminides 1 were prepared as previously described. ${ }^{3}$

Synthesis of Complexes 4. General Procedure. To a solution of the aminide $1(1 \mathrm{mmol})$ in $\mathrm{CH}_{2} \mathrm{Cl}_{2}(5 \mathrm{~mL})$ under argon a $1 \mathrm{M}$ solution of $\mathrm{ZnCl}_{2}(1 \mathrm{~mL})$ in $\mathrm{Et}_{2} \mathrm{O}$ was added. The white precipitate formed was filtered off, washed with $\mathrm{Et}_{2} \mathrm{O}$ and recrystallized to afford pure complexes 4.

Dichlorobis (1-cinnamoylamino-l-pyridinium) Zinc(II) (4a). Yield: $65 \% . \mathrm{Mp} \mathrm{248-250}{ }^{\circ} \mathrm{C}(\mathrm{EtOH}) ; \mathrm{IR}(\mathrm{KBr})$ $\mathrm{v}_{\max } 3108,3032,1648,1603,1473 \mathrm{~cm}^{-1},{ }^{1} \mathrm{H}-\mathrm{NMR}\left(300 \mathrm{Mhz}, \mathrm{DMSO}-\mathrm{d}_{6}\right) \delta 8.79$ (dd, 2H, J=6.2 and J=1.2 Hz), 8.15-8.21 (m, 1H), 7.8-7.9 (m, 2H), 7.5-7.6 (m, 2H), 7.3-7.4 (m, 4H), $6.78(\mathrm{~d}, 1 \mathrm{H}, \mathrm{J}=15.8 \mathrm{~Hz}) \mathrm{ppm}$; MS (70 eV) $m z$ (rel. int.): 224 (57), 223 (100), 121 (34), 102 (29), 79 (51), 77 (25). Anal. Calcd for $\mathrm{C}_{28} \mathrm{H}_{24} \mathrm{Cl}_{2} \mathrm{~N}_{4} \mathrm{O}_{2} \mathrm{Zn}: \mathrm{C}$, $57.51, \mathrm{H}, 4.24, \mathrm{~N}, 9.58$. Found: $\mathrm{C}, 57.46 ; \mathrm{H}, 4.13 ; \mathrm{N}, 9.62$.

Dichlorobis(I-benzoylamino-l-pyridinium) Zinc(II) (4b). Yield: $85 \%$. Mp $195-196^{\circ} \mathrm{C}(\mathrm{EtOH})$; IR (KBr) $\mathrm{v}_{\max }$ $3076,2923,1682.1594,1563 \mathrm{~cm}^{-1},{ }^{1} \mathrm{H}-\mathrm{NMR}\left(300 \mathrm{MHz}\right.$, DMSO-d $\left.\mathrm{d}_{6}\right) \delta 8.84(\mathrm{dd}, 2 \mathrm{H}, \mathrm{J}=5.6$ and J=1.0 Hz), $8.1-8.2$ 
(m, IH), 7.9-8.0 (m, 2H), 7.3-7.4 (m, 3H) ppm; MS (70 eV) m/z (rel int.) 198 (40), 197 (92), 121 (100), 79 (79), 77 (78), 51 (43). Anal. Calcd for $\mathrm{C}_{24} \mathrm{H}_{20} \mathrm{Cl}_{2} \mathrm{~N}_{4} \mathrm{O} Z \mathrm{Zn}: \mathrm{C}, 54.11 ; \mathrm{H}, 3.78 ; \mathrm{N}, 10.52$. Found: $\mathrm{C}, 54.02 ; \mathrm{H}, 3.53 ; \mathrm{N}, 10.30$

Dichlorobis(2-cinnamoylamino-2-isoquinolinium) Zinc(II) (4c). Yield: $95 \% . \mathrm{Mp} 268-270^{\circ} \mathrm{C}\left(\mathrm{CH}_{3} \mathrm{CN}\right)$ $\operatorname{IR}(\mathrm{KBr}) \cup_{\max } 3028,1646,1603,1469,1380 \mathrm{~cm}^{-1},{ }^{1} \mathrm{H}-\mathrm{NMR}\left(300 \mathrm{MHz}, \mathrm{DMSO}-\mathrm{d}_{6}\right) \delta: 9.91(\mathrm{~s}, 1 \mathrm{H}), 8.44(\mathrm{dd}, 1 \mathrm{H}$. $\mathrm{J}=7.0$ and $\mathrm{J}=2.4 \mathrm{~Hz}), 8.35(\mathrm{~d}, 1 \mathrm{H}, \mathrm{J}=8.3 \mathrm{~Hz}), 8.31(\mathrm{~d}, 1 \mathrm{H}, \mathrm{J}=7.0 \mathrm{~Hz}), 8.21(\mathrm{~d}, 1 \mathrm{H}, \mathrm{J}=8.1 \mathrm{~Hz}), 8.0-8.1(\mathrm{~m} . \mathrm{H}), 7.8-$ $7.9(\mathrm{~m}, \mathrm{IH}), 7.5-7.6(\mathrm{~m}, 2 \mathrm{H}), 7.2-7.4(\mathrm{~m}, 3 \mathrm{H}), 7.43(\mathrm{~d}, 1 \mathrm{H}, \mathrm{J}=15.9 \mathrm{~Hz}) \mathrm{ppm}$; MS (70 eV) $m z z$ (rel. int ) : $274(54)$. $273(86), 130(21), 129$ (100), 102 (34). Anal Calcd for $\mathrm{C}_{36} \mathrm{H}_{28} \mathrm{Cl}_{2} \mathrm{~N}_{4} \mathrm{O}_{2} \mathrm{Zn}: \mathrm{C}, 63.13 ; \mathrm{H}, 4.12 ; \mathrm{N}, 818$ Found $\mathrm{C}$. $6252 . \mathrm{H}, 4.36 ; \mathrm{N}, 8.24$

I) ichlorobis(2-benzoylamino-2-isoquinolinium) Zinc(II) (4d). Yield: 70\%. Mp 219-220 ${ }^{\circ} \mathrm{C}(\mathrm{EtOH}) ; \mathrm{IR}(\mathrm{KBr})$ $v_{\max } 3046,1684,1669,1599,1503 \mathrm{~cm}^{-1}, 1 \mathrm{H}-\mathrm{NMR}\left(300 \mathrm{MHz}, \mathrm{DMSO}-\mathrm{d}_{6}\right) \delta: 10.27(\mathrm{~s}, 1 \mathrm{H}), 8.84(\mathrm{~d}, 1 \mathrm{H}, \mathrm{J}=6.9 \mathrm{~Hz})$, $8.66(\mathrm{~d}, 1 \mathrm{H}, \mathrm{J}=6.9 \mathrm{~Hz}), 8.53(\mathrm{~d}, 1 \mathrm{H}, \mathrm{J}=8.4 \mathrm{~Hz}), 8.40(\mathrm{~d}, 1 \mathrm{H}, \mathrm{J}=8.4 \mathrm{~Hz}), 8.2-8.3(\mathrm{~m}, \mathrm{lH}), 8.0-8.1(\mathrm{~m}, 3 \mathrm{H}) .7 .5-7.7$ (m, 3H) ppm. MS (70 eV) $m z$ (rel. int.) $247(56), 129(35), 105$ (45), 77 (100), 51 (51). Anal. Calcd for $\mathrm{C}_{31} \mathrm{H}_{24} \mathrm{Cl}_{2} \mathrm{~N}_{4} \mathrm{O} 2 \mathrm{Zn}: \mathrm{C}, 63.73 ; \mathrm{H}, 3.82 ; \mathrm{N}, 8.85$. Found: $\mathrm{C}, 63.68 ; \mathrm{H}, 3.54 ; \mathrm{N}, 887$.

Jichlorohis(I-cinnamoylamino-3-methyl-3H-l-imidazolium) Zinc(II) (4e). Yield: $70 \%$. Mp 245-246 ${ }^{\circ} \mathrm{C}$ (EtOH); IR (KBr) $\cup_{\max } 3143,1642,1616,1576,1449 \mathrm{~cm}^{-1},{ }^{1} \mathrm{H}-\mathrm{NMR}$ ( $300 \mathrm{MHz}$, DMSO-d $\left.{ }_{6}\right) \delta: 925$ (s, IH), $74-$ $7.5(\mathrm{~m}, 4 \mathrm{H}), 7.2-7.3(\mathrm{~m}, 4 \mathrm{H}), 7.03(\mathrm{~d}, 1 \mathrm{H}, \mathrm{J}=16.14 \mathrm{~Hz}), 3.80(\mathrm{~s}, 3 \mathrm{H})$. MS (70 eV) $m z$ (rel. int ) $227(22), 226(92)$, $195(56), 149(72), 91$ (38), $77(58), 57$ (100). Anal. Calcd for $\mathrm{C}_{26} \mathrm{H}_{26} \mathrm{Cl}_{2} \mathrm{~N}_{6} \mathrm{O}_{2} \mathrm{Zn}: \mathrm{C}, 52.28 ; \mathrm{H}, 4.43, \mathrm{~N}, 14.22$ Found $\mathrm{C}, 52.55, \mathrm{H}, 4.50, \mathrm{~N}, 14.15$

1)ichlorobis( l-benzoylamino-3-methyl-3H-l-imidazolium) Zinc(II) (4f). Yield $92 \%$. Mp $>290{ }^{\circ} \mathrm{C}(\mathrm{dec})$ : IR $(\mathrm{KBr}) \cup_{\max } 3146,1692.1591,1554,1552,1484 \mathrm{~cm}^{-1},{ }^{1} \mathrm{H}-\mathrm{NMR}\left(300 \mathrm{MHz}, \mathrm{DMSO}-\mathrm{d}_{6}\right) \delta: 9.65(\mathrm{~s}, 1 \mathrm{H}), 7.9-8.0$ $(\mathrm{m}, 2 \mathrm{H}), 7.64(\mathrm{~s}, \mathrm{lH}), 7.48(\mathrm{~s}, \mathrm{lH}), 7.2-7.3(\mathrm{~m}, 3 \mathrm{H}), 3.8(\mathrm{~s}, 3 \mathrm{H}) \mathrm{ppm}$; MS (70 eV) $\mathrm{mz}$ (rel int ) 201 (100), 200 (88), $124(99), 105(60), 103(44), 82(55), 77$ (89), 51 (43). Anal Calcd for $\mathrm{C}_{22} \mathrm{H}_{22} \mathrm{Cl}_{2} \mathrm{~N}_{6} \mathrm{O} Z \mathrm{Zn}: \mathrm{C}, 49.05, \mathrm{H}$. $412:$ N. 15 60. Found $\mathrm{C}, 48.84 ; \mathrm{H}, 4.17 ; \mathrm{N}, 15.45$.

1-Thiocinnamoylpyridinium $\mathrm{N}$-aminide (5a). To a suspension of $\mathrm{N}$-cinnamoylpyridinium iodide $(0.35 \mathrm{~g}, 1$ mmol) in $\mathrm{MeCN}(15 \mathrm{~mL}) 0^{\circ} \mathrm{C}$ cooled was added $\mathrm{P}_{4} \mathrm{~S}_{10}(0.44 \mathrm{~g} 1 \mathrm{mmol})$ and $\mathrm{Et}_{3} \mathrm{~N}(0.56 \mathrm{~mL} .4 \mathrm{mmol})$. The mixture was stirred at room temperature for 24 and then water $(20 \mathrm{~mL})$ was added. The solution was extracted with $\mathrm{CH}_{2} \mathrm{Cl}_{2}$ (4x35 mL) and the organic phase was separated and dried over $\mathrm{MgSO}_{4}$. After elimination of the solvent under reduced pressure the residue obtained was chromatographed on silica gel. Elution with EtOH afforded the title compound (95 mg, 39\%) as a dark yellow solid. Mp. 115-117 ${ }^{\circ} \mathrm{C}$ (EtOAc); IR (KBr) $\mathrm{v}_{\max } 3031,1628,1612.1572$. $1470,1381,1175 \mathrm{~cm}^{-1},{ }^{1} \mathrm{H}$ NMR (300 MHz, DMSO-d6) 8.63 (dd, $2 \mathrm{H}, \mathrm{J}=6.8$ and $\left.\mathrm{J}=1.2 \mathrm{~Hz}\right), 8.30(\mathrm{t}, 1 \mathrm{H}, \mathrm{J}=7.8 \mathrm{~Hz}$ ), $7.9-8.0(\mathrm{~m}, 2 \mathrm{H}), 7.75(\mathrm{~d}, 1 \mathrm{H}, \mathrm{J}=15.5 \mathrm{~Hz}), 7.6-7.7(\mathrm{~m}, 2 \mathrm{H}), 7.3-7.4(\mathrm{~m}, 3 \mathrm{H}), 7.10(\mathrm{~d}, 1 \mathrm{H}, \mathrm{J}=15.5 \mathrm{~Hz}) \mathrm{ppm}$. MS $(70$ eV) $m=$ (rel. int) 160 (24), $129(100), 128(25), 102(38), 79(42)$ Anal. Calcd for $\mathrm{C}_{14} \mathrm{H}_{12} \mathrm{~N}_{2} \mathrm{~S}: \mathrm{C}, 69.97, \mathrm{H}, 5.03$; N. 11.66, S. 13.34 Found: C, 69.62; H, 4.95; N. $11.43 ;$ S, 13.15

Synthesis of Bis-aminides 6. (jeneral Procedure. To a mixture of $0.44 \mathrm{~g}(2 \mathrm{mmol})$ of $\mathrm{N}$-aminopyridinium iodide, $1.1 \mathrm{~g}(8 \mathrm{mmol})$ of $\mathrm{K}_{2} \mathrm{CO}_{3}$ and $\mathrm{CH}_{2} \mathrm{Cl}_{2}(20 \mathrm{~mL})$ was slowly added $1 \mathrm{mmol}$ of the corresponding diacyl chloride in $\mathrm{CH}_{2} \mathrm{Cl}_{2}(15 \mathrm{~mL})$. The reaction mixture was stirred at room temperature for $24 \mathrm{~h}$. The inorganic precipitate was separated by filtration and washed with $\mathrm{CH}_{2} \mathrm{Cl}_{2}(2 \times 5 \mathrm{~mL})$. The filtrate and washes were concentrated under reduced pressure and the oily residue was triturated with EtOAc. The solid thus obtained was isolated by filtration and purified by recrystallization to afford pure bis-aminides 6 . 
N-N-(1,7-Diaza-2,6-dioxohepta-1,7-diyl)bis-1-pyridinium Diaminide (6a). Yield: $70 \%$. Mp 176-178 ${ }^{\circ} \mathrm{C}$ (Acetone/CH $\mathrm{CH}_{3} \mathrm{CN}$, white powder); IR (KBr) $v_{\max } 3112,1616,1554,1337,1049 \mathrm{~cm}^{-1} ;{ }^{1} \mathrm{H}-\mathrm{NMR}$ (300 MHz, DMSO$\left.d_{6}\right) \delta 8.63(\mathrm{~d}, 4 \mathrm{H}, \mathrm{J}=6.2 \mathrm{~Hz}), 8.06(\mathrm{t}, 2 \mathrm{H}, \mathrm{J}=7.5 \mathrm{~Hz}), 7.79(\mathrm{dd}, 4 \mathrm{H}, \mathrm{J}=7.5$ and $\mathrm{J}=6.2 \mathrm{~Hz}), 2.08(\mathrm{t}, 4 \mathrm{H}, \mathrm{J}=6.6 \mathrm{~Hz})$, $1.86(\mathrm{t}, 2 \mathrm{H}, \mathrm{J}=6.6 \mathrm{~Hz}) \mathrm{ppm}$; Anal. Calcd for $\mathrm{C}_{15} \mathrm{H}_{16} \mathrm{~N}_{4} \mathrm{O}_{2}: \mathrm{C}, 63.37 ; \mathrm{H}, 5.67 ; \mathrm{N}, 19.71$. Found: $\mathrm{C}, 63.23 ; \mathrm{H}, 5.73$; N. 19.92

$N, N$-(1,8-Diaza-2,7-dioxoocta-1,8-diyl)bis-1-pyridinium Diaminide (6b). Yield: $81 \% . \quad$ Mp 174-176 ${ }^{\circ} \mathrm{C}$ (Acetone/ $\mathrm{CH}_{3} \mathrm{CN}$, white powder); IR (KBr) $v_{\max } 3058,2944,1620,1577,1554,1381,1080 \mathrm{~cm}^{-1} .{ }^{1} \mathrm{H}-\mathrm{NMR}(300$ $\left.\mathrm{MHz}, \mathrm{DMSO}-\mathrm{d}_{6}\right) \delta 8.64(\mathrm{~d}, 4 \mathrm{H}, \mathrm{J}=6.6 \mathrm{~Hz}), 8.05(\mathrm{t}, 2 \mathrm{H}, \mathrm{J}=7.0 \mathrm{~Hz}), 7.78(\mathrm{dd}, 4 \mathrm{H}, \mathrm{J}=7.0$ and $\mathrm{J}=6.6 \mathrm{~Hz}), 2.0-2,1(\mathrm{~m}$, 4H), 1.5-1.6 (m, 4H) ppm; MS (70 eV) m/z (rel. int ) 298 (1), 177 (22), 163 (12), 135 (18), 121 (100), 79 (93) Anal Calcd for $\mathrm{C}_{16} \mathrm{H}_{18} \mathrm{~N}_{4} \mathrm{O}_{2} \cdot \mathrm{H}_{2} \mathrm{O}: \mathrm{C}, 60.75 \mathrm{H}, 6.37 ; \mathrm{N}, 17.71$. Found: $\mathrm{C}, 60.68 ; \mathrm{H}, 5.88 ; \mathrm{N}, 17.66$.

N.N'-(1,10-Diaza-2,9-dioxodeca-1,10-diyl)bis-1-pyridinium Diaminide (6c).Yield: $94 \% . \mathrm{Mp} 163-165{ }^{\circ} \mathrm{C}$ $\left(\mathrm{CH}_{3} \mathrm{CN}\right.$. brown powder); IR (KBr) $\mathrm{U}_{\max } 3106,2933,1618,1574,1560,1373,1091 \mathrm{~cm}^{-1}:{ }^{1} \mathrm{H}-\mathrm{NMR}(300 \mathrm{MHz}$. DMSO- $\left._{\mathrm{G}}\right) \delta 8.62(\mathrm{dd}, 4 \mathrm{H}, \mathrm{J}=6.3$ and $\mathrm{J}=1.2 \mathrm{~Hz}), 8.06(\mathrm{~m}, 2 \mathrm{H}), 7.78(\mathrm{dd}, 4 \mathrm{H}, \mathrm{J}=8.2$ and $\mathrm{J}=6.3 \mathrm{~Hz}), 2.06(\mathrm{t}, 4 \mathrm{H}$. $\mathrm{J}=7.5 \mathrm{~Hz}), 1.5-1.6(\mathrm{~m}, 4 \mathrm{H}), 1.3-1.4(\mathrm{~m}, 4 \mathrm{H}) \mathrm{ppm} ; \mathrm{MS}(70 \mathrm{eV}) \mathrm{m} / \mathrm{z}$ (rel. int) $163(3), 149(10), 135(32), 121(84)$, 93 (3), 79 (100). Anal. Calcd for $\mathrm{C}_{18} \mathrm{H}_{22} \mathrm{~N}_{4} \mathrm{O}_{2}: \mathrm{C}, 66.24 ; \mathrm{H}, 6.79 ; \mathrm{N}, 17.17$. Found: $\mathrm{C}, 65.85 ; \mathrm{H}, 6.85 ; \mathrm{N}, 16.77$.

$N, N-\left(1,12\right.$-diaza-2,11-dioxododeca-1,12-diyl)bis-1-pyridinim Diaminide (6d). Yield: $70 \%$. Mp 105 $110^{\circ} \mathrm{C}$ (AcOEt/Acetone. pale yellow powder); IR (KBr) $v_{\max } 3116,2923,1667,1618,1544,1098 \mathrm{~cm}^{-1},{ }^{1} \mathrm{H}-\mathrm{NMR}(300$ $\left.\mathrm{MHz}, \mathrm{DMSO}-\mathrm{d}_{6}\right) \delta 8.62(\mathrm{~d}, 4 \mathrm{H}, \mathrm{J}=6.7 \mathrm{~Hz}), 8.05(\mathrm{t}, 2 \mathrm{H}, \mathrm{J}=7.5 \mathrm{~Hz}), 7.78(\mathrm{dd}, 4 \mathrm{H}, \mathrm{J}=7.5$ and $\mathrm{J}=6.7 \mathrm{~Hz}), 2.07(\mathrm{t}, 4 \mathrm{H}$, $\mathrm{J}=7.0 \mathrm{~Hz}), 1.5-1.6(\mathrm{~m}, 4 \mathrm{H}), 1.2-1.3(\mathrm{~m}, 8 \mathrm{H}) \mathrm{ppm}$; MS (70 eV) $\mathrm{m} / \mathrm{z}$ (rel. int.) $177(4), 121(69), 93(10), 79(100)$, 56 (29). Anal. Calcd for $\mathrm{C}_{20} \mathrm{H}_{26} \mathrm{~N}_{4} \mathrm{O}_{2}: \mathrm{C}, 67.77 ; \mathrm{H}, 7.39 ; \mathrm{N}, 15.81$. Found: $\mathrm{C}, 67.89 ; \mathrm{H}, 7.46 ; \mathrm{N}, 15.93$

N.N (1.14-diaza-2,13-dioxotetradeca-1,14-diyl)bis-1-pyridinium Diamide (6e). Yield: 89\%. Mp $92-94{ }^{\circ} \mathrm{C}$ (EtOAc, white powder); IR (Kbr) $v_{\max } 3116,2922,1668,1618,1548,1374,1096 \mathrm{~cm}^{-1}$; ${ }^{1} \mathrm{H}-\mathrm{NMR}$ (300 MHz, DMSO- $\left._{6}\right) \delta: 8.62(\mathrm{dd}, 4 \mathrm{H}, \mathrm{J}=6.8$ and $\mathrm{J}=1.3 \mathrm{~Hz}), 8.07(\mathrm{t}, 2 \mathrm{H}, \mathrm{J}=7.7 \mathrm{~Hz}), 7.80(\mathrm{dd}, 4 \mathrm{H}, \mathrm{J}=7.4$ and $\mathrm{J}=6.8 \mathrm{~Hz}), 2.05$ $(\mathrm{t}, 4 \mathrm{H}, \mathrm{J}=7.1 \mathrm{~Hz}), 1.5-1.6(\mathrm{~m}, 4 \mathrm{H}), 1.2-1.3(\mathrm{~m}, 12 \mathrm{H}) \mathrm{ppm}$. Anal. Calcd for $\mathrm{C}_{22} \mathrm{H}_{30} \mathrm{~N}_{4} \mathrm{O}_{2}: \mathrm{C}, 69.08: \mathrm{H}: 7.91 ; \mathrm{N}, 14.65$ Found: C. $68.95 ; \mathrm{H}, 8.02 ; \mathrm{N}, 14.60$

1,1-Terphthaloilimino-bis-1-pyridinium Diaminide (6f). Yield: $22 \%$. Mp $275-277^{\circ} \mathrm{C}(\mathrm{EtOH}$, white powder): IR (KBr) $v_{\max } 3064,2944,1549,1466,1335,1301,1250,1179 \mathrm{~cm}^{-1},{ }^{1} \mathrm{H}-\mathrm{NMR}$ (CD OD, 300 $\mathrm{MHz}): \delta 8.7-8.8(\mathrm{~m}, 4 \mathrm{H}), 8.2-8.3(\mathrm{~m}, 2 \mathrm{H}), 8.10(\mathrm{~s}, 4 \mathrm{H}), 7.9-8.0(\mathrm{~m}, 4 \mathrm{H}) \mathrm{ppm}$. Anal. Calcd for $\mathrm{C}_{18} \mathrm{H}_{1+} \mathrm{N}_{4} \mathrm{O}_{2}$ C, $67.92 ; \mathrm{H}, 4.43 ; \mathrm{N}, 17.60$. Found: C, $67.89 ; \mathrm{H}, 4.39 ; \mathrm{N}, 17.43$

Synthesis of complexes 7. General procedure. To a solution of 6 (1 mmol) in $\mathrm{CH}_{2} \mathrm{Cl}_{2}(5 \mathrm{~mL})$ a $1 \mathrm{M}$ solution of $\mathrm{ZnCl}_{2}(1 \mathrm{~mL})$ in $\mathrm{Et}_{2} \mathrm{O}$ was added at room temperature. The white precipitate which immediately formed was isolated by filtration and recrystallized to give pure complexes 7

Dichloro/N,N-(1,7-diaza-2,6-dioxohepta-1,7-diyl)bis-1-pyridinium] Zinc(II) (7a).Yield: 85\%. Mp 313-314 ${ }^{\circ} \mathrm{C}(\mathrm{MeOH}) ; \mathrm{IR}(\mathrm{KBr}) \cup_{\max } 3114,2944,1622,1584,1390 \mathrm{~cm}^{-1},{ }^{\prime} \mathrm{H}-\mathrm{NMR}\left(300 \mathrm{MHz}, \mathrm{DMSO}-\mathrm{q}_{6}\right) \delta 8.65$ (d, 4H, $\mathrm{J}=6.0 \mathrm{~Hz}), 8.34(\mathrm{t}, 2 \mathrm{H}, \mathrm{J}=7.5 \mathrm{~Hz}), 7.98(\mathrm{dd}, 4 \mathrm{H}, \mathrm{J}=7.5$ and $\mathrm{J}=6.0 \mathrm{~Hz}), 2.41(\mathrm{t}, 4 \mathrm{H}, \mathrm{J}=6.7 \mathrm{~Hz}), 1.86(\mathrm{q}, 2 \mathrm{H}, \mathrm{J}=7.5$ $\mathrm{Hz}$ ) ppm. Anal. Calcd for $\mathrm{C}_{15} \mathrm{H}_{16} \mathrm{Cl}_{2} \mathrm{~N}_{4} \mathrm{O}_{2} \mathrm{Zn} . \mathrm{H}_{2} \mathrm{O}: \mathrm{C}, 41.08 ; \mathrm{H}, 4.14 ; \mathrm{N}, 12.77$. Found : $\mathrm{C}, 41.23 ; \mathrm{H}, 4.03 ; \mathrm{N}$. 12.65 .

Dichloro[N,N-(1,8-diaza-2,7-dioxoocta-1,8-diyl)bis-1-pyridinium] Zinc(II) (7b). Yield: 80\%. Mp 309-311 ${ }^{\circ} \mathrm{C}(\mathrm{MeOH}) ; \mathbb{R}(\mathrm{KBr}) v_{\max } 3113,2925,1621,1609,1560,1374 \mathrm{~cm}^{-1},{ }^{1} \mathrm{H}-\mathrm{NMR}\left(300 \mathrm{MHz}\right.$, DMSO-d $\left.\mathrm{d}_{6}\right) 88.63$ (d, $4 \mathrm{H}, \mathrm{J}=6.7 \mathrm{~Hz}), 8.09(\mathrm{t}, 2 \mathrm{H}, \mathrm{J}=7.5 \mathrm{~Hz}), 7.81(\mathrm{dd}, 4 \mathrm{H}, \mathrm{J}=7.5$ and $\mathrm{J}=6.7 \mathrm{~Hz}), 2.1-2.2(\mathrm{~m}, 4 \mathrm{H}), 1.60-1.65(\mathrm{~m}, 4 \mathrm{H}) \mathrm{ppm}$. 
Positive FAB-MS $m / z$ (rel. int.) 435 (1), 399 (12), 299 (100), 95 (33). Anal. Calcd for $\mathrm{C}_{16} \mathrm{H}_{18} \mathrm{Cl}_{2} \mathrm{~N}_{4} \mathrm{O}_{2} \mathrm{Zn} \cdot \mathrm{H}_{2} \mathrm{O}: \mathrm{C}$, 42.46; $\mathrm{H}, 4.45 ; \mathrm{N}, 12.38$. Found: $\mathrm{C}, 42.23 ; \mathrm{H}, 4.62 ; \mathrm{N}, 11.95$.

Dichloro/N,N'-(I,10-diaza-2,9-dioxodeca-1,10-diyl)bis-1-pyridinium/ Zinc(II) (7c) Yield: 89\%. Mp 303-305 ${ }^{\circ} \mathrm{C}(\mathrm{MeOH})$; IR (KBr) $v_{\max } 3114,2936,1622,1586,1554,1398 \mathrm{~cm}^{-1} .{ }^{1} \mathrm{H}-\mathrm{NMR}\left(300 \mathrm{MHz}\right.$. DMSO-d $\left.\mathrm{d}_{6}\right) \delta 8.60(\mathrm{~d}$ $4 \mathrm{H}, \mathrm{J}=6.7 \mathrm{~Hz}), 8.13(\mathrm{t}, 2 \mathrm{H}, \mathrm{J}=7.3 \mathrm{~Hz}), 7.82(\mathrm{dd}, 4 \mathrm{H}, \mathrm{J}=7.3$ and $\mathrm{J}=6.7 \mathrm{~Hz}), 2.14(\mathrm{t}, 4 \mathrm{H}, \mathrm{J}=7.5 \mathrm{~Hz}), 1.5-1.6(\mathrm{~m}, 4 \mathrm{H})$ 1. 2-1.3 (m, 4H) ppm. Positive FAB-MS $m z$ (rel. int.) 463 (9), 427 (67), 327 (100), 95 (59). Anal. Calcd for $\mathrm{C}_{18} \mathrm{H}_{22} \mathrm{Cl}_{2} \mathrm{~N}_{4} \mathrm{O}_{2} \mathrm{Zn} . \mathrm{H}_{2} \mathrm{O}: \mathrm{C}, 44.98 ; \mathrm{H}, 5.03 ; \mathrm{N}, 11.06$. Found: C, 45.11; H, 4.83: N, 11.25

Dichloro/ $N, N^{\prime}-(1,12$-diaza-2,11-dioxododeca-1,12-diyl)his-1-pyridinium/ Zinc(II) (7d). Yield $90 \%$ Mp 300-301 ${ }^{\circ} \mathrm{C}(\mathrm{MeOH}), \mathrm{IR}(\mathrm{KBr}) \cup_{\max } 3117,2923,1624,1591.1554 \mathrm{~cm}^{-1} .{ }^{1} \mathrm{H}-\mathrm{NMR}\left(300 \mathrm{MHz}\right.$. DMSO-d $\left.\mathrm{d}_{6}\right) \delta 859$ $(\mathrm{d}, 4 \mathrm{H}, \mathrm{J}=6.3 \mathrm{~Hz}), 8.14(\mathrm{t}, 2 \mathrm{H}, \mathrm{J}=7.4 \mathrm{~Hz}), 7.84(\mathrm{dd}, 4 \mathrm{H}, \mathrm{J}=7.4$ and $\mathrm{J}=6.3 \mathrm{~Hz}), 2.14(\mathrm{t}, 4 \mathrm{H}, \mathrm{J}=7.5 \mathrm{~Hz}), 1.5-1.6(\mathrm{~m}$ $4 \mathrm{H}), 1.2-1.3(\mathrm{~m}, 8 \mathrm{H}) \mathrm{ppm}$. Anal. Calcd for $\mathrm{C}_{20} \mathrm{H}_{26} \mathrm{Cl}_{2} \mathrm{~N}_{4} \mathrm{O}_{2} \mathrm{Zn} \cdot \mathrm{H}_{2} \mathrm{O}: \mathrm{C}, 47.22 ; \mathrm{H}, 5.55 ; \mathrm{N}, 11.01$. Found $\mathrm{C}, 7.35$; H. $5.31, N, 10.87$

Dichloro/N.N-11.14-diaza-2,13-dioxotetradeca-1,14-diyl)his-1-pyridinium/Zinc(II) (7e). Yield: $95 \% \mathrm{Mp}$ $140-142{ }^{\circ} \mathrm{C}(\mathrm{EtOH}) ; \mathrm{IR}(\mathrm{KBr}) \mathrm{v}_{\max } 3111,2922,3848,1620,1586,1556,1398,1080 \mathrm{~cm}^{-1} ;{ }^{1} \mathrm{H}-\mathrm{NMR}(300 \mathrm{MHz}$. DMSO-d $\left.\mathrm{d}_{6}\right) \delta 8.58(\mathrm{~d}, 4 \mathrm{H}, \mathrm{J}=6.0 \mathrm{~Hz}), 8.17(\mathrm{t}, 2 \mathrm{H} . \mathrm{J}=7.5 \mathrm{~Hz}), 7.85(\mathrm{dd}, 4 \mathrm{H}, \mathrm{J}=7.5$ and $6.0 \mathrm{~Hz}), 2.15(\mathrm{t}, 4 \mathrm{H}, \mathrm{J}=7.3$ $\mathrm{Hz}$ ). 1.5-1.6 (m, 4H), 1.2-1.3 (m, 12H) ppm. Positive FAB-MS $m$ z (rel. int.) 519 (2), 483 (36), 383 (78), 95 ( ( 100$)$ Anal Calcd for $\mathrm{C}_{22} \mathrm{H}_{310} \mathrm{Cl}_{2} \mathrm{~N}_{4} \mathrm{O}_{2} \mathrm{Zn} . \mathrm{H}_{2} \mathrm{O}: \mathrm{C}, 49.22 ; \mathrm{H}, 6.01 ; \mathrm{N}, 10.44$. Found: $\mathrm{C}, 49.45 ; \mathrm{H}, 6.28 ; \mathrm{N}, 10.29$

N.N-(2.15-1)iaza-3,1f-dioxohexadeca-2,15-diyl)his-1-pyridinium Diodide (9). A mixture of $6 \mathrm{e}(0.38 \mathrm{~g}, 1$ mmol) and methyl iodide $(0.56 \mathrm{~g}, 4 \mathrm{mmol})$ in acetone $(10 \mathrm{~mL})$ was refluxed for $18 \mathrm{~h}$. The precipitate formed was isolated by filtration and recrystallized from acetone/EtOH to yield $0.62 \mathrm{~g}(95 \%)$ of a yellow powder. Mp 187-188 ${ }^{\circ} \mathrm{C}, \mathrm{IR}(\mathrm{KBr}) \mathrm{u}_{\text {mux }} 3111,3009,2921,1671,1549,1467,1377,1288 \mathrm{~cm}^{-1},{ }^{1} \mathrm{H}-\mathrm{NMR}\left(300 \mathrm{MHz}, \mathrm{DMSO}-\mathrm{d}_{6}\right)$ ) $: 9.23$ (dd, $2 \mathrm{H}, \mathrm{J}=6.9$ and $\mathrm{J}=1.2 \mathrm{~Hz}), 8.80(\mathrm{t}, 2 \mathrm{H}, \mathrm{J}=7.7 \mathrm{~Hz}), 8.34(\mathrm{~m}, 4 \mathrm{H}), 2.07(\mathrm{~m}, 4 \mathrm{H}), 1.55-1.59(\mathrm{~m}, 4 \mathrm{H}), 1.24-1.3 .3$ $(\mathrm{m}, 12 \mathrm{H}) \mathrm{ppm}$. Anal Calcd for $\mathrm{C}_{24} \mathrm{H}_{36} \mathrm{I}_{2} \mathrm{~N}_{4} \mathrm{O}_{2}: \mathrm{C}, 42.98 ; \mathrm{H}, 5.56: \mathrm{N}, 8.67$. Found: $\mathrm{C}, 43.26 ; \mathrm{H}, 5.45 ; \mathrm{N}, 8.41$

Single-( rystal X-Ray Structure Determination of ('omplex 4a. Intensity data were collected using a CAD4 diffractometer with graphite-monochromated $\mathrm{MoK} \alpha$ radiation $(0.71069 \AA)$. Data were collected at room temperature. Intensities were corrected for Lorenz and polarization effects in the usual manner. No absorption or extinction corrections were made.

The structure was solved by a combination of direct methods and Fourier synthesis and refined (on F) by full matrix least-squares calculations. All the non hydrogen atoms were anisotroplically refined. In the last cycle of refinement hydrogen atoms were introduced from geometrical positions and fixed with thermal parameters equivalent to those of the carbons to which they were attached. Final values of $R=0.072$ and $R w=0098$ with $R w=\left[\sum\left[w\left|F_{0}\right|-\left|F_{c}\right|\right]^{2} / w\left[\mid F_{0}{ }^{2}\right]^{2} / 2\right.$ and $w=4 F_{0}^{2} /\left[\sigma\left|F_{0}\right|\right]^{2}$ obtained

Anomalous dispersion corrections and atomic scattering factors were taken from International Tables.". Calculations were performed with the SDP package on a Microvax II computer. ${ }^{1.3}$

Acknowledgment. The authors acknowledge the Comisió Interdepartamental de Recerça $i$ Innovació Tecnologica (CIRIT, project QFN94-4619) for financial support and the Ministerio de Educación y Ciencia for a studentship (M.A.H.) 


\section{References}

1. For reviews of N-aminides, see: (a) Timpe, H. J. Adv. Heterocycl. Chem., 1974, 17, 213. (b)Y. Tamura, Y.; Ikeda, M. Adv. Heterocycl. Chem, 1981, 29, 71. (c) Ollis, W. D.; Stanforth, S. P. Tetrahedron, 1993, 41, 714.

2. Martinez, Y.; Heras, M. A.; Vaquero, J. J.; García Navio, J. L.; Alvarez-Builla, J. Tetrahedron Lett. 1995, 36,8513

3. Heras, M. A.; Molina, A.; Vaquero, J. J.; García Navio J. L.; Alvarez-Builla, J. J. Org. Chem. 1993, 58, 5862.

4. Heras, M. A.; Vaquero, J. J.; Garcia Navio, J. L.; Alvarez-Builla, J. Tetrahedron, 1996, 45, 14297

5. Heras, M. A.; Vaquero, J. J.; Garcia Navio, J. L.; Alvarez-Builla, J. J. Org. Chem. 1996, 61, 9009.

6. Dias, S. A.; Downs, A. W.; McWhinnie, W. R. J. (hem. Soc., 1974, 162.

7. Chia, L. Y.; Dias, S. A.; McWinnie, W. R. J. Inorg. Nucl. Chem. 1975, 38, 1263

8. Bhakagrahi, D. Heterocycles, 1982, 19, 11.

9. Heras, M. A.; Vaquero, J. J.; García Navio, J. L.; Alvarez-Builla, J. Tetrahedron Lett. 1995, 36, 455.

10. Rebek, J.; Trend, J. E.; Wattley, R. V.; Chakravorty, S. J. Am. Chem. Soc. 1979, 101, 4333.

11. Cuevas, J.-C.; Mendoza, J.; Prados, P.; Hérnandez-Cano, F.; Foces-Foces, C. J. (hem. Soc. ('hem. Comm. 1986, 1641 .

12. International Tables for X-Ray Crystallography, vol IV, Kynoch, Birmighan, 1974.

13. B. A. Frenz and Associates, Inc. SDP. Texas A\&M and Enraf-Nonius. College Station Tx 77840 and Delft, 1985

(Received in UK 30 January 1997; revised 18 March 1997; accepted 20 March 1997) 